\title{
HEAT AND MASS TRANSFER UNDER AN INFANT RADIANT WARMER - DEVELOPMENT OF A NUMERICAL MODEL
}

\author{
Anna M. Fic ${ }^{1}$, Derek B. Ingham ${ }^{2}$, Maciej K. Ginalski ${ }^{3}$, Andrzej J. Nowak ${ }^{4}$ and Luiz Wrobel ${ }^{5}$ \\ ${ }^{1}$ Centre for CFD, University of Leeds, LS2 9JT, UK \\ A.M.Fic@leeds.ac.uk \\ ${ }^{2}$ Centre for CFD, University of Leeds \\ D.B.Ingham@leeds.ac.uk \\ ${ }^{3}$ ANSYS UK \\ maciej.ginalski@ansys.com \\ ${ }^{4}$ Silesian University of Technology \\ andrzej.j.nowak@polsl.pl \\ ${ }^{5}$ Brunel University \\ Luiz.Wrobel@brunel.ac.uk
}

\begin{abstract}
The main objectives of this paper are to present a procedure of how to create and set up a model for the physical processes that take place within an infant radiant warmer and to validate that Computational Fluid Dynamics (CFD) can be used to resolve such problems. In this study, the results are obtained for a simplified model, both in terms of the geometry employed and the prescribed boundary conditions. The results were numerically verified in terms of the convergence history, monitor data and the physical correctness. This study shows that the physical situation is unsteady and the results tend to oscillate, almost periodically, around a mean value. The results presented in the paper are found to be in qualitative agreement with the experimental data. This gives us confidence that the techniques employed in this paper are appropriate and form the starting point for the inclusion of more realistic effects, e.g. real shape of the newborn and radiant lamp, heat generated inside the newborn, moisture transport, etc.
\end{abstract}

Key Words: Computational Fluid Dynamics, Heat Transfer, Natural Convection, Radiant Warmer, Neonatology

\section{Introduction}

Survival of each newborn depends on the ability for it to regulate its body temperature. While full-term and healthy neonates (newborns) do not have serious problems with thermoregulation, preterm and sick babies often are not able to keep their body temperature at a constant level without any external assistance. Without the environment in which a preterm neonate can maintain a normal body temperature $\left(\sim 37^{\circ} \mathrm{C}\right)[1]$, it will risk cold stress and hypothermia, which may cause an increase in the morbidity and mortality $[2,3]$. This can develop very quickly because a premature baby can receive up to $120 \mathrm{kcal} \mathrm{kg}^{-1} \mathrm{day}^{-1}[4,5]$ and a wet newborn at birth looses around $200 \mathrm{kcal} \mathrm{kg}^{-1} \mathrm{~min}^{-1}$ [5]. Obviously, the normal clinical situation does not include such high heat losses, but they are still substantial enough to overwhelm the heat production capabilities of the newborn. 
There are three general ways to keep newborns warm and these include incubators, radiant warmers and heated mattresses. The first two are in common use in hospitals. The incubators were invented earlier than radiant warmers and they provide an enclosed environment with warm air circulating inside the device. Radiant warmers are open devices. They consist of a radiant heater placed above a neonate lying on a crib. In this case, a child is heated by a source of radiant heat. The power of this source is servo-controlled from the skin temperature [6]. The main advantage of these devices over incubators is the ease in the access to the baby, thus enabling various medical interventions. However, there are also a few concerns when using radiant warmers. The main drawback is that they increase evaporative heat losses from the newborn body [7]. This is due to the fact that premature babies have a very thin and permeable skin and this, combined with a strong radiation source, yields high evaporative heat losses through the skin. This situation is likely to result in serious dehydration in the case of very premature babies and it was just this problem that generated the cooperation between Hammersmith Hospital in London with the University of Leeds and Brunel University. Therefore, the main goal of this cooperation is to find a solution for the problem of the dehydration of babies nursed under radiant warmers. Once we have a fully developed model, with the heat generation within the baby and the mass transfer incorporated, then using this sophisticated modelling approach we will verify some of the alternative warming ideas which would not yield such high evaporative heat losses in order to improve the situation when babies being nursed under radiant warmers become dehydrated. This work will be focused on babies being nursed in the post-delivery rooms, where the neonates are dry and rarely dressed.

Computational Fluid Dynamics (CFD) applied to the living organisms can provide a better understanding of the interaction of a human body with the surrounding environment. As one of the branches of fluid mechanics, CFD uses numerical methods and algorithms to solve and analyze problems that involve mass and heat transfer processes. Therefore, all the processes occurring between the human body and its environment can be resolved. Starting from conduction, convection and radiation, through evaporation from the skin and ending on the respiration, all these processes can be successfully modelled and resolved. An example of such use of CFD is shown in the previous work of a co-author where he has performed an extensive study on the heat and mass transfer processes in an infant incubator, see [8].

A mathematical and computational model of a neonate under a radiant warmer, that incorporates the heat and mass transfer processes, is developed in order to provide a better understanding of how a radiant heat source interacts with a newborn. Once this has been achieved, a more sophisticated model that includes heat generation inside a neonate and moisture transport will be developed. The aim of this paper is to present a procedure of how to create and set up a model for the presented problem. For this reason, the model has been simplified, both in terms of the employed geometry and the prescribed boundary conditions.

\section{Mathematical model}

Each CFD package requires a rigorous theoretical framework. This framework consists of the conservation equations of mass, energy, momentum and, for reacting flows, conservation of species. Moreover, additional transport equations are solved for turbulent flows. 
The following assumptions have been made for the model:

- The physical setup of the problem is in steady state. However, transient calculations were performed to improve the rate of convergence of the results and to reach the steady state solution.

- The heat source is modelled in the form of a flat surface with a prescribed constant heat transfer rate; this is a simplification that will be reconsidered in the future models.

- A constant skin temperature is prescribed to the model of the neonate, however in the future research the heat generation inside a baby will be considered.

- The moisture transport is neglected, however due to the fact that it will have a significant impact on the results, it will be taken into account in the future research.

- For the purpose of this investigation, the geometry of the baby is simplified to one half of a circular cylinder of finite length.

- Due to the symmetry of the problem under investigation, only one quarter of the physical domain is considered.

- Based on the calculation of the Rayleigh number for this particular physical situation, it is assumed that the flow is turbulent. The Reynolds averaging processes were chosen to resolve the turbulent fluid flow. This means that the Reynolds-averaging is performed on the momentum equation. The averaging process consists of decomposing the flow variables into their mean and fluctuating components. The $k-\varepsilon$ RNG model is used for modelling the turbulence, based on the fact that $k-\varepsilon$ models have an option to enable the resolution of buoyant flows and the RNG model is effective for both high and low Re number turbulent flows. The natural convection phenomenon present in the model considered in this paper is characterized by relatively low velocities, which in some regions might results in low $\mathrm{Re}$ turbulent flow, thus the use of the $k-\varepsilon$ RNG model. The Enhanced Wall Treatment is used to calculate the flow in the near wall regions.

- The air density is calculated assuming that the air is an incompressible ideal gas, which is a good assumption for problems with natural convection and where there are no significant pressure variations. The Boussinesq model is not appropriate for the cases with high gradients of the temperature, which appear near the heat source in the considered model.

- The medium between the radiant warmer and the neonate is assumed to be dry air and thus it is transparent for the thermal radiation. For radiation modelling, the Discrete Ordinates (DO) model has been chosen, because it is the only model in FLUENT that can calculate specular reflection, and this will be crucial in the future models, due to the presence of parabolic reflectors surrounding the cylindrical heat source. Various settings for the angular discretization of the domain have been tested and we have found quantitatively that the results did not change significantly when increasing the angular discretization. Therefore, for the purpose of this study, the value of $4 \times 3$ has been assumed to be sufficient.

- All the surfaces present in the model are treated as grey bodies.

Once the proposed method has been validated then all these assumptions will be relaxed in more sophisticated mathematical models that will be developed in the future. 


\subsection{Transport equations}

Mass, momentum and energy transfer calculations are based on the following conservation equations, respectively [9]:

$$
\begin{gathered}
\frac{\partial \rho}{\partial t}+\frac{\partial}{\partial x_{i}}\left(\rho w_{i}\right)=0 \\
\frac{\partial}{\partial t}\left(\rho w_{j}\right)+\frac{\partial}{\partial x_{i}}\left(\rho w_{i} w_{j}\right)=\rho g_{j}-\frac{\partial p}{\partial x_{j}}+\frac{\partial}{\partial x_{i}}\left(\mu \frac{\partial w_{j}}{\partial x_{i}}\right) \\
\frac{\partial}{\partial t}(\rho c T)+\frac{\partial}{\partial x_{i}}\left(\rho w_{i} c T\right)=\frac{\partial}{\partial x_{i}}\left(\lambda \frac{\partial T}{\partial x_{i}}\right)
\end{gathered}
$$

In the equations above, Einstein notation is used, and the subscripts $i, j=1,2,3$ denote, respectively, the directions $x_{1}, x_{2}$ and $x_{3} ; \rho$ is the density of the fluid $\left(\mathrm{kg} \mathrm{m}^{-3}\right), t$ is time (s), $w$ stands for the velocity vector $\left(\mathrm{m} \mathrm{s}^{-1}\right), g$ is gravitational acceleration $\left(\mathrm{m} \mathrm{s}^{-2}\right), p$ is pressure $\left(\mathrm{N} \mathrm{m}^{-2}\right), \mu$ is dynamic viscosity $\left(\mathrm{N} \mathrm{s} \mathrm{m}^{-2}\right), c$ is specific heat $\left(\mathrm{J} \mathrm{kg}^{-1} \mathrm{~K}^{-1}\right), \lambda$ stands for thermal conductivity $\left(\mathrm{W} \mathrm{m}{ }^{-1}\right.$ $\mathrm{K}^{-1}$ ) and $T$ is the temperature (K).

We now need to add two equations for calculating the turbulence. The $k-\varepsilon$ models are based on the transport equations for the turbulence kinetic energy, $k\left(\mathrm{~m}^{2} \mathrm{~s}^{-2}\right)$, and its dissipation rate, $\varepsilon\left(\mathrm{m}^{2} \mathrm{~s}^{-3}\right)$. While $k$ determines the energy of the turbulence, $\varepsilon$ is an indication of the scale of the turbulence. The viscosity in Eqn. (2.2) is in fact a sum of the turbulent and laminar viscosities. Therefore, these two additional equations are necessary to determine the turbulent viscosity, which is proportional to $k$ and $\varepsilon$. The equations for $k$ and $\varepsilon$ are given by [10]:

$$
\begin{gathered}
\rho \frac{D k}{D t}=P+\frac{\partial}{\partial x_{i}}\left(\alpha_{k} \mu_{e f f} \frac{\partial k}{\partial x_{i}}\right)-\rho \varepsilon \\
\rho \frac{D \varepsilon}{D t}=\frac{\partial}{\partial x_{i}}\left(\alpha_{\varepsilon} \mu_{e f f} \frac{\partial \varepsilon}{\partial x_{i}}\right)+\frac{\varepsilon}{k}\left(C_{1 \varepsilon}-C_{2 \varepsilon} \rho \varepsilon\right)+C_{3 \varepsilon} \rho \varepsilon \nabla \cdot U-\rho R
\end{gathered}
$$

Where: $\alpha_{k}$ and $\alpha_{\varepsilon}$ are the inverse effective Prandtl number for $k$ and $\varepsilon$, respectively and $C_{1 \varepsilon}, C_{2 \varepsilon}, C_{3 \varepsilon}$ are the empirical constants in the equation for the turbulent dissipation rate. Further details on the components of Eqns. (2.4) - (2.5) are given by [10].

The radiative transfer equation (RTE) for an absorbing, emitting, and scattering medium at the position $\boldsymbol{r}$ and in the direction $\boldsymbol{s}$ takes the following form [11]:

$$
\hat{\boldsymbol{s}} \cdot \nabla I(\boldsymbol{r}, \hat{\boldsymbol{s}})=\alpha(\boldsymbol{r}) I_{b}(\boldsymbol{r})+\beta(\boldsymbol{r}) I(\boldsymbol{r}, \hat{\boldsymbol{s}})+\frac{\sigma_{s}(\boldsymbol{r})}{4 \pi} \int_{0}^{4 \pi} d I\left(\boldsymbol{r}, \hat{\boldsymbol{s}}^{\prime}\right) \Phi\left(\boldsymbol{r}, \hat{\boldsymbol{s}}, \hat{\boldsymbol{s}}^{\prime}\right) d \Omega^{\prime}
$$

Where: $\hat{\boldsymbol{s}}$ is the direction vector, $I$ is the radiation intensity $\left(\mathrm{W} \mathrm{m}^{-2} \mathrm{sr}^{-1}\right), \boldsymbol{r}$ is the position vector; $\alpha$ is the absorption coefficient $\left(\mathrm{m}^{-1}\right), I_{b}$ is the blackbody intensity $\left(\mathrm{W} \mathrm{m}^{-2} \mathrm{sr}^{-1}\right), \beta$ stands for the extinction coefficient $\left(\mathrm{m}^{-1}\right), \sigma_{s}$ is the scattering coefficient $\left(\mathrm{m}^{-1}\right), \hat{s}^{\prime}$ is the scattering direction vector, $\Phi$ stands for the scattering phase function $\left(\mathrm{sr}^{-1}\right)$ and $\Omega^{\prime}$ is the solid angle (sr) 


\subsection{Geometry and mesh}

Before proceeding into a detailed description of the computational model, some aspects need further explanation. First of all, the dimensions of the model of the radiant warmer described in this paper are based on the information found in [12] for a KDC 660 type of infant radiant warmer. Recently, cooperation with Hammersmith Hospital in London has been initiated and this hospital is equipped with Fisher \& Paykel CosyCot infant radiant warmers. As a result of this cooperation, the temperature measurements on a CosyCot radiant warmer, both with and without a baby inside, were taken at the hospital using a Flir thermal camera. Moreover, Fisher \& Paykel supplied us with some data on the emissivity of the surfaces composing the warmer as well as the temperatures of the radiating element inside the lamp. Because of the substantial differences in the geometry of the KDC and CosyCot radiant warmers, it was necessary to consider the geometry of the CosyCot in the developed model. This was essential in order to be able to compare the numerical results with the experimental data. Thus, there are two separate models: the initial model that was built based on the dimensions of the KDC radiant warmer and the second model that was created based on the dimensions provided by Fisher \& Paykel. The future research will consider only CosyCot radiant warmer because only for this radiant warmer we have experimental data against which we can validate our model. It is also worth noting that we have performed several benchmark tests on our CFD software and validated our computational procedures.

The geometry of a real baby is very complex and detailed. In order to capture all the details of the body, a very fine mesh is required. Such a grid requires over one million elements, see Fig. 2.1. Because of the time consuming calculations that such a fine mesh requires, for the purposes of this study a simplified model has been built. Instead of the real baby geometry, one half of a cylinder is used with a length of $0.4 \mathrm{~m}$, which is the length of a typical baby, and the radius $0.071 \mathrm{~m}$, which gives a comparable surface area to that of a neonate. This simplification will enable us to create coarse meshes for relatively fast simulations. This is due to the fact that before we move to more complex and detailed modelling, we have to confirm that our mathematical and computational approach is appropriate and accurate. The best way to gain this confidence is to start the modelling with something simple, both in terms of the geometry, mesh and boundary conditions. Such a model can be used to compute various simulations with different case setups in order to find the effects of the physical behaviour on the results. Therefore the set of results presented in this paper is used for observing the behaviour of the natural convection phenomena combined with a radiant heat source. In fact, this stage is one of the most important ones in CFD.

The radiant warmer is an open device and has no external walls that could create the boundaries for the numerical model. Therefore, the domain had to be artificially limited. The artificial boundaries were imposed on the model to limit the physical size of the dimensions of the computational domain. The domain consists of four main parts. There are several surfaces that represent the inlets and one that represents the outlet, which, together with models of the bed and infant, close the solution domain. The fourth part of the model is the radiant warmer that is modelled in the form of a cuboid.

The whole domain, before considering the symmetry planes, is presented in Fig. 2.2. The results presented in this paper have been obtained for the domain with the dimensions of $1.6 \times 1.2 \times 1.6 \mathrm{~m}$ (length $\times$ width $\times$ height, respectively). Other sizes of the domain have also been investigated and it was found that when a smaller domain was considered then the air flow around the radiant warmer created recirculation zones that go beyond the imposed inlet boundaries. As a result of that test an expanded domain, presented in Fig. 2.2, has been built in order to avoid this situation. However, 
when taking a larger domain the results obtained in the vicinity of the model of the neonate were almost indistinguishable. Therefore we conclude that the results presented in this paper will adequately model the conditions in which the radiant warmer will be operated. In practise the room in which the radiant warmer is placed will be larger than the dimensions considered in the presented model.

As already mentioned, the dimensions of the radiant warmer and the mattress, as well as the position of the heat source with respect to the mattress, are all based on the dimensions of the KDC 660 radiant warmer, that were found in [12]. Therefore the size of the mattress is $0.8 \times 0.4 \mathrm{~m}$ and the radiant warmer has the dimensions of $0.3 \times 0.2 \times 0.05 \mathrm{~m}$. The radiant warmer is placed $0.65 \mathrm{~m}$ over the mattress. The height of the bed was set to be $0.3 \mathrm{~m}$. This height is not the total height of the bed used in the radiant warmers. The bottom surface around the bed in the model is not a floor, but an inlet. In the previously tested different domain sizes, the bottom inlet was at the level of the mattress. However, for the purposes of this paper, the bottom inlet has been moved downwards in order to decrease the influence of the inlet boundaries on the convective plume.

A set of tetrahedral grids has been employed and they consist of both tetrahedral and wedge-shaped elements. The last ones were used for the mesh generation within the boundary layer. The number of elements within those grids is presented in Table 2.1. A grid consisting of 90k elements is shown in Fig. 2.3. The size of the elements changes within the domain, from the smallest elements placed around the cylinder and radiant warmer, where a boundary layer has also been created.

\subsection{Boundary conditions}

The boundary conditions are schematically presented in Fig. 2.3. Pressure inlet and pressure outlet boundary conditions have been prescribed at the boundaries of the computational model with no gauge pressure. This yields the movement of the fluid solely via natural convection. The operating density has been set to be $1.177 \mathrm{~kg} \mathrm{~m}^{-3}$, which is the density of the air entering the domain. The thermal properties of the air used for the calculations are given in Table 2.2. The other surfaces, such as the radiant warmer, mattress, bed and baby, have a wall boundary condition prescribed. The temperature on the baby's skin is assumed to be constant and equal to $37{ }^{\circ} \mathrm{C}$. This is another simplification that has been introduced to the model. In reality, the temperature of the baby varies significantly and the purpose of using radiant warmers is to maintain the infant in a certain temperature range. The aim of prescribing a constant temperature boundary condition to the skin is to avoid creating a model that would be too complex at this stage and, therefore might have difficulties with convergence during the calculations.

Both the bed and the mattress, on which the baby is placed, are assumed to be insulated. This assumption is based on the fact that these mattresses are designed to reduce the heat losses through conduction from the baby. Therefore this is a realistic first approximation, but this condition will be reconsidered in more sophisticated models that are being developed. The side and top walls of the radiant warmer are assumed to be at the same temperature as the air entering the domain, namely $27^{\circ} \mathrm{C}$. The bottom surface of the warmer has a heat transfer rate prescribed equal to $600 \mathrm{~W}$ [12]. In reality, the sources of the heat in the radiant warmers vary in terms of their geometry. The heat source can be either split into a few bulbs placed in different positions inside the radiant lamp, or it can be directed to one or more cylindrical, long tube-shaped bulbs. 
The emissivity of the skin and the heat source are set to 0.95 , and the emissivities of all the other surfaces are assumed to be equal to 0.9 . The values of the emissivity prescribed for the heat source and the mattress are based on the information obtained from the producer of the Fisher \& Paykel CosyCot radiant warmers. The diffuse fraction for all the surfaces in the model is set equal to 1, and this means that these surfaces are assumed to reflect the radiation diffusively.

\section{Results and discussion}

In this section we present some of the results for the cases investigated. In order to avoid divergence of the results during the iterative process, the calculations are performed in a particular manner. The calculation procedure is presented in Fig. 3.1. The first two steps represent the situation where only natural convection from the baby is considered. There is no heat source prescribed on the radiant warmer and no radiation is modelled. In the third step, the radiation is turned on and in the fourth step, the heat source is switched on by prescribing the heat transfer rate at the radiant warmer to be equal to $600 \mathrm{~W}$.

There are many factors that can significantly influence the results, for example the operating density, which has to be set correctly in order to obtain reasonable results. The operating density is a parameter that contributes to the value and direction of the buoyancy force that causes the movement in the natural convection. Following a series of tests, this value has been set equal to $1.177 \mathrm{~kg} \mathrm{~m}^{-3}$.

The first observation to be made while inspecting the results is that they are unstable and it is difficult to obtain convergence. In order to improve the rate of convergence, transient calculations were performed to reach the steady state solution. These problems are caused by the nature of the natural convection phenomena, which is not easy to overcome. Changes in both the heat transfer rate on the cylinder and the temperatures on the mattress for several different meshes are presented in Fig. 3.2 as a function of the time step. The time step used for the calculations presented in this paper is $\Delta t=0.1 \mathrm{~s}$ and it has been chosen after a series of tests involving smaller and larger time steps. It can be concluded from Fig. 3.2 that both quantities plotted oscillate within relatively small amplitude around a constant value. Therefore, these constants are also shown in Fig. 3.2. In fact, this is what occurs in the reality, as natural convection is not a stable phenomenon and thus some oscillations in the predicted results may be expected.

The results presented in Fig. 3.2 are also used for the purpose of testing the grid independency of the solution. Fig. 3.2(a) presents the average heat transfer rate values on the cylinder during the calculations. All the values of the heat transfer rate have a negative sign, which indicates that the surface gains heat rather than releasing it to the surrounding air. Clearly this is in agreement with what we would physically expect, because the purpose of using the radiant warmer is to provide more heat gain than what is being lost to the environment. The negative sign in the heat transfer rate also means that all the heat losses from the baby due to convection and radiation are compensated by the radiant heat source.

In terms of the average heat transfer rate on the cylinder, the grid independency test presented in Fig. 3.2(a) shows that there is a decreasing tendency when moving from crude to fine meshes. It can also be observed that the three cases with the finest mesh (case 2, 3 and 4) give very similar results for the average total surface heat transfer rate. The difference between the heat transfer rate value obtained for cases 2 and 4 is less than $3 \%$. 
The temperatures shown in Fig. 3.2(b) do not appear to have the same stable tendency as for the total surface heat transfer rate. The highest values of the average temperatures on the mattress were obtained for the crudest mesh. Then, there is a significant gap between the results obtained for the temperatures in the case 0 and case 3 . This might suggest that case 0 gives the least satisfactory results and should not be used in any further investigation process. Case 1 gives the average temperature that is closest to the one with the finest mesh. However, the difference between the results obtained in case 1 and case 2 is about $0.1{ }^{\circ} \mathrm{C}$, i.e. an error which is about $0.02 \%$. This implies that the results obtained for case 2 may be successfully considered as mesh independent. For this reason, all the results shown further in this paper are those obtained for case 2 .

The temperature fields and velocity vectors resulting from these simulations, plotted for convenience on the symmetry planes, are presented in Fig. 3.3. It can be observed that in the central part of the domain, above the baby, a convective plume is created with higher temperatures and velocities. The air enters the domain through the bottom and side inlets. Next, the air is warmed by the baby's skin and flows up towards the radiant warmer, where another portion of the heat is absorbed by the air from the hot bottom surface of the warmer, and thus creating a hot plume above it. The warm air exits the domain through the top outlet. The air around the radiant warmer is much warmer than the maximum value of the temperature in the scale for Fig. 3.3 would suggest. The maximum air temperatures in the domain will reach the value of the heat source temperature, shown in Fig. 3.4. Further, the temperature scale presented in Fig. 3.3 has been adjusted in order to better visualize the convective plume.

The situation with high temperatures above the radiant warmer is caused by the fact that the source of heat is here prescribed to the flat surface. Therefore, there are significant heat losses to the air in the convective plume. In reality, the heat source is in the form of a bulb that is surrounded by reflective surfaces and thus the convective losses from the source of the heat probably are not that significant. However, it should be noted, that in a real radiant lamp, there are significant heat losses from the lamp cover. This is due to the fact that a bulb placed inside the lamp heats the reflecting surfaces that surround such a bulb and that heat is further conducted to the (plastic) casing. This situation has been observed during the measurements performed at the Hammersmith Hospital. It was observed that the temperature of the lamp cover can reach up to $70^{\circ} \mathrm{C}$. This indicates that the warm air plume, which is observed in Fig. 3.3, should also be observed in reality, but for different reasons.

A balance of the heat transfer rates on the major surfaces in the model is presented in Table 3.1. All the quantities presented in this table are valid for one quarter of the full model. The difference between the total surface heat transfer rate and the radiation heat transfer rate is the contribution from the convective heat losses. It can easily be observed that the total surface heat transfer rate for the baby is lower than the radiation heat transfer rate. This is due to the fact that the only heat gains for a baby nursed under a radiant warmer are the gains due to radiation. Therefore the $3 \mathrm{~W}$ of the total surface heat transfer rate for a baby, that are presented in Table 3.1, have to be divided into the gains that will not be lost $(2.6 \mathrm{~W})$ but transferred further to the body and losses due to convection $(0.4 \mathrm{~W})$. If it concerns the radiant warmer, then the total surface heat transfer rate is the value prescribed in the boundary conditions for the heat source $(600 \mathrm{~W})$. It can be noticed that only a part of the heat is being transferred via radiation, the remaining part are the losses due to convection. The total surface heat transfer rate for the mattress is $0 \mathrm{~W}$, due to the fact that in the present model the mattress was assumed to be insulated. 
The amount of radiant heat that reaches the mattress leads to the situation that relatively high temperatures are generated on the mattress, as shown in Fig. 3.4(a). During the experimental measurements that have been performed at the Hammersmith Hospital, the average temperatures on the mattress registered by the camera were in the vicinity of $50^{\circ} \mathrm{C}$, while the results of the numerical investigation indicate that the average temperature is of the order of $52^{\circ} \mathrm{C}$. This shows a good agreement of numerical results with the measurements.

Modelling the radiant source in the form of a flat surface leads to a diffusive emission of radiation. A radiating element in the real lamp also emits radiation diffusively. However, in reality it is surrounded by the reflecting surfaces that return the rays of the radiant energy in a mirror-like manner. The reflectors have a specially designed curvature, so that most of the reflected radiant energy could be directed towards the baby. Due to the fact that in the described model radiation is evenly emitted in all directions, a significant part of that energy is lost as it reaches the external boundaries. The importance of that situation is presented in Table 3.2. Taking into account that the radiation heat transfer rate emitted from the surface with the heat transfer rate prescribed is $120 \mathrm{~W}$ for a quarter of the model, the level of the escaping energy contributes to most of that heat transfer rate. The baby, on the other hand, only gains about $2.5 \%$ of the radiant energy emitted by the heat source.

Fig. 3.4(b) shows the temperature distribution on the surface with the heat source prescribed. The distribution is quite uniform, with the maximum temperature from the scale on almost the whole surface. Even though these temperatures are high, they are not as high as in reality. In a real lamp, the heating element with a $100 \%$ heating power, may reach temperatures as high as $680^{\circ} \mathrm{C}$. This information, obtained from the producer of the Fisher \& Paykel CosyCot radiant warmer, has been confirmed during the thermographic measurements at the Hammersmith Hospital. However, it should be noted that the two temperatures of the heating elements, both in the model and in the real lamp, are not entirely comparable. This is due to the fact that the heat transfer rate in both cases is prescribed to the surfaces of different areas. The area of the heating surface in the model is higher than the area of the heating element surface in the real lamp. This implies a logical conclusion that when the same value of the heat transfer rate is prescribed to two surfaces of different areas, then lower temperatures will be generated at the larger surface, while on the smaller surface higher temperatures would be observed.

\section{Conclusions}

The main goal of this project is to find a solution for the problem of neonates becoming dehydrated when being nursed under radiant warmers. The first step towards achieving this goal was to create a simplified model in order to ensure that the results are stable and reasonably accurate. In this paper, the physical phenomenon that occurs under a radiant warmer for such a simplified model has been investigated. It has been observed that within the obtained results there is good qualitative agreement with the experimental data. For example, the average temperature of the mattress obtained from the experiments and the numerical results vary only in $2{ }^{\circ} \mathrm{C}$. Thus the results obtained by this simplified model give us confidence that the techniques employed in this paper are appropriate and form the starting point for including more realistic effects, e.g. real shape of the newborn, heat generated inside the newborn, moisture transport, etc.

It has also been observed that the results are sensitive to changes made in the model. For instance, while resolving the natural convection phenomena, the calculation of density is very important. In 
this case, the air is assumed to be an incompressible ideal gas, and therefore the density becomes solely temperature dependent. This assumption can be made in the situation when the pressure changes are relatively small, and thus it can be applied in the model considered in this paper.

A remedy to overcome the relatively high temperatures predicted at the mattress may be to create a solid representation of the mattress with a finite depth. This would transmit the heat that is now accumulated at the surface to the inside of the mattress and thus lead to a decrease in the temperature. Further improvements in the results may be obtained by introducing a more detailed geometry of the lamp with the radiating element surrounded by the reflecting parabolic surfaces. A mirror-like reflection of the radiation from the reflecting surfaces will direct more radiant energy towards the baby, while in the model described in this paper, the radiation is emitted in all directions diffusively. Therefore, some of the radiant warmer energy is lost, because radiation escapes from the domain. Moreover, the influence of the humidity in the air should be investigated, because this may have some effect on the results when a high radiant heat source is considered.

\section{Acknowledgments}

The authors would like to express their appreciation to the external advisors of this project, namely Professor Andrzej J. Nowak from the Silesian University of Technology, Poland, and Professor Luiz Wrobel from Brunel University. We also acknowledge the great assistance of Dr Denis Azzopardi from Hammersmith Hospital, London, and his support on this project.

Moreover, a word of appreciation should go to the European Union for the Marie Curie Fellowship programme as well as to the Centre for CFD in University of Leeds for enabling the work on the project described in this paper.

The authors also appreciate the help of Fisher \& Paykel in supplying the necessary data for the calculations

\section{References}

[1] Boxwell G. 'Neonatal Intensive Care Nursing', London: Routledge 2000, Chapter 4.

[2] Knobel R, Holditch-Davis D. Thermoregulation and heat loss prevention after birth during neonatal intensive care unit stabilization of extremely low-birth weight infants. Journal of Obstetric, Gynecologic and Neonatal Nursing 2007, 36: 280-287.

[3] Lyon A. temperature control in the newborn infant. Current Paediatrics 2006, 16: 386-392.

[4] Hulzebos CV, Sauer PJJ. Energy requirements. Seminars in Fetal and Neonatal Medicine 2007, 12: 2-10.

[5] Nalepka CD. Understanding thermoregulation in newborns. Journal of Obstetric, Gynecologic and Neonatal Nursing 1976, 5: 17-19.

[6] Wheldon AE, Rutter N. The heat balance of small babies nursed in incubators and under radiant warmers. Early Human Development 1982, 6: 131-143

[7] Bell EF. Infant incubators and radiant warmers. Early Human Development 1983, 8: 351-375.

[8] Ginalski MK, Nowak AJ and Wrobel L. A combined study of heat and mass transfer in an infant incubator with an overhead screen. Medical Engineering and Physics 2007, 29: 531-541.

[9] Versteeg HK, Malalasekera W. 'Computational Fluid Dynamics. The finite volume method', New York: Longman 1995. 
[10] Papageorgakis GC, Assanis DN. Comparison of linear and nonlinear RNG-based k-epsilon models for incompressible turbulent flows. Numerical Heat Transfer, Part B 1999, 35:1-22.

[11] Modest MF. 'Radiative Heat Transfer', Amsterdam, Boston: Academic Press 2003.

[12] LeBlanc MH, Edwards NK. Artifacts in the measurement of skin temperature under infant radiant warmer. Annals of Biomedical Engineering 1985, 13: 443-450 
(a)

(b)
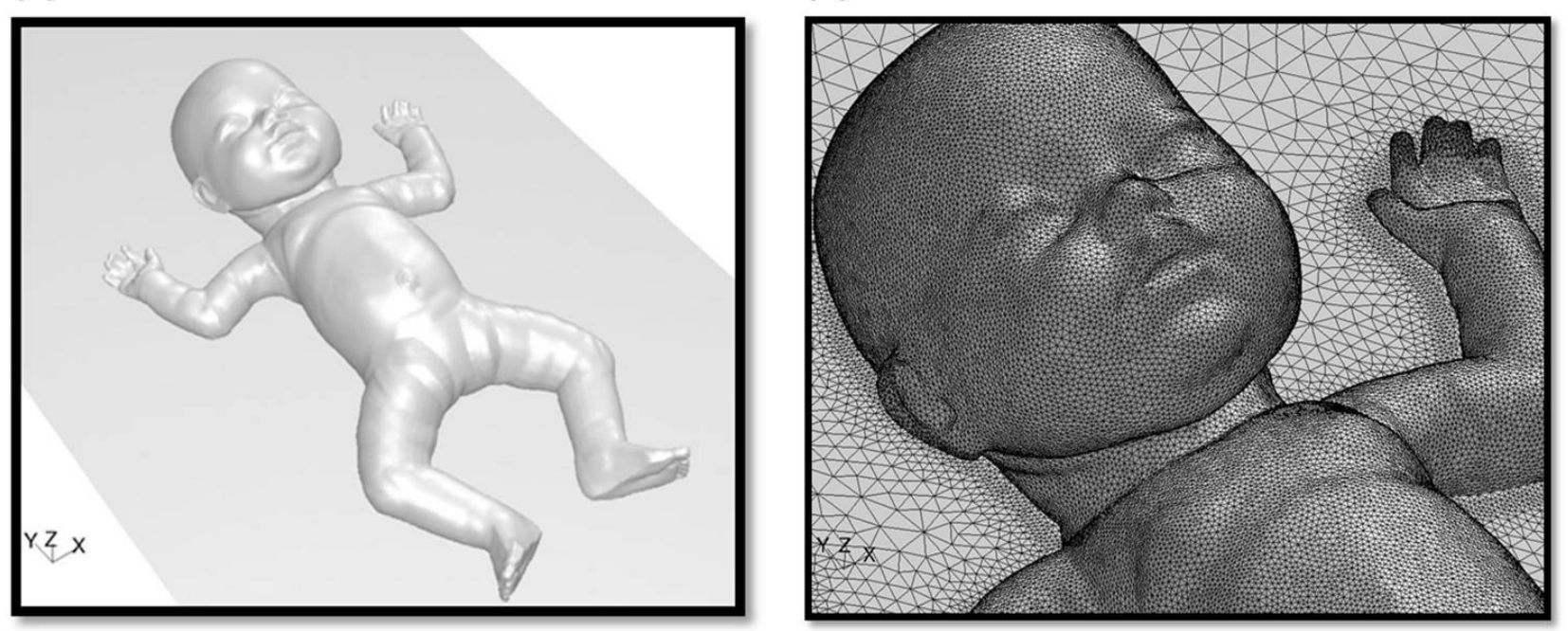

Figure 2.1. (a) The geometry of the baby, and (b) the mesh employed.

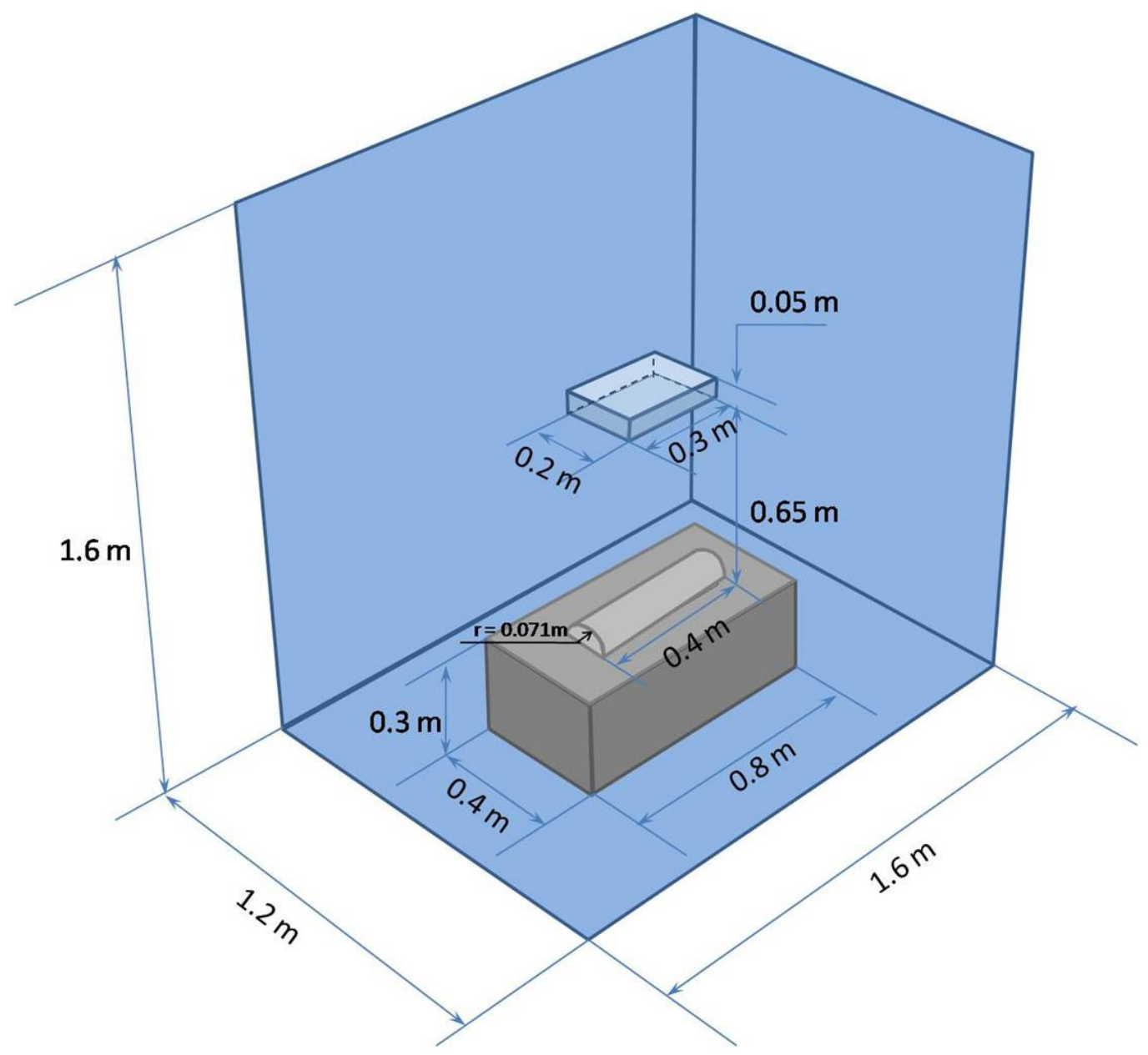

Figure 2.2. The whole computational domain with the dimensions. 
(a)

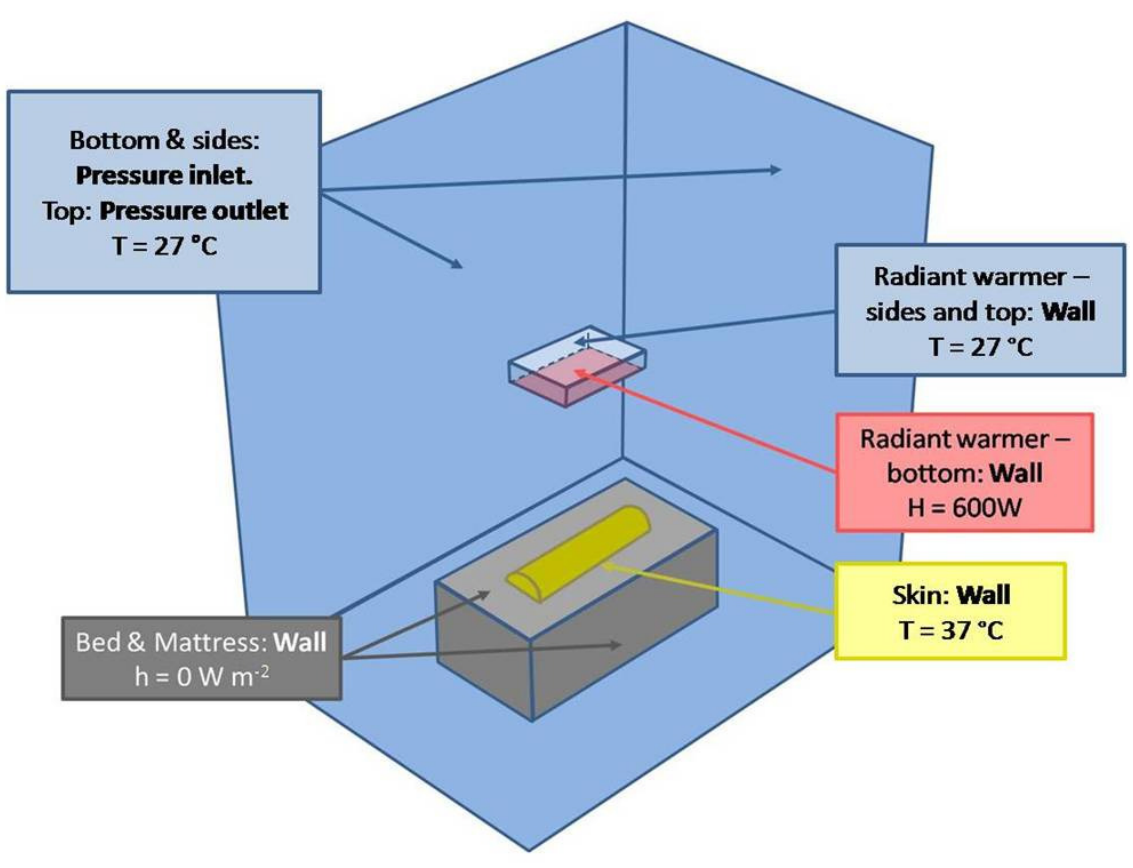

(b)

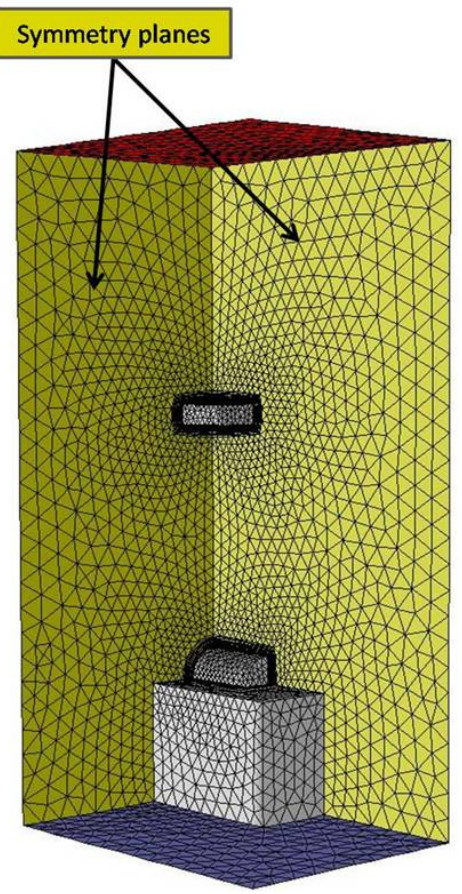

Figure 2.3. (a) Schematic diagram of the geometry and boundary conditions, and (b) the model with the created tetrahedral grid and symmetry planes considered.

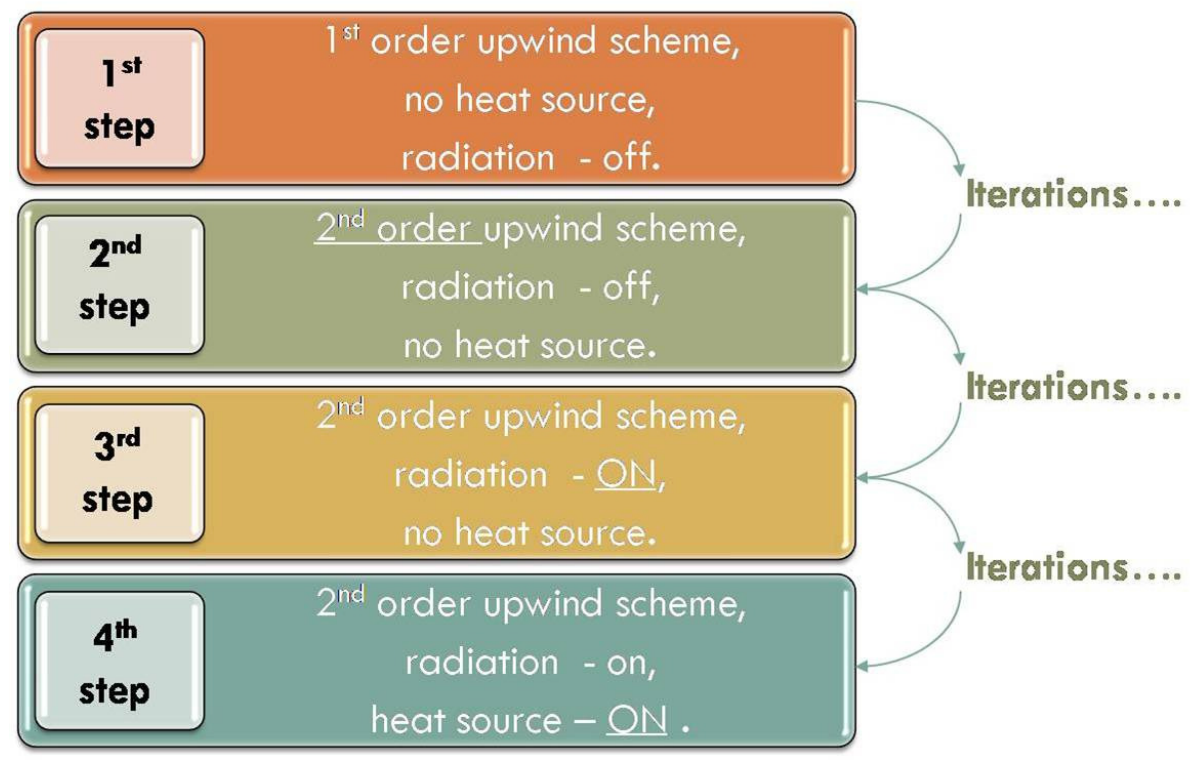

Figure 3.1. Calculation procedure. 
(a) Changes of the average heat flux on infant's skin during calculations

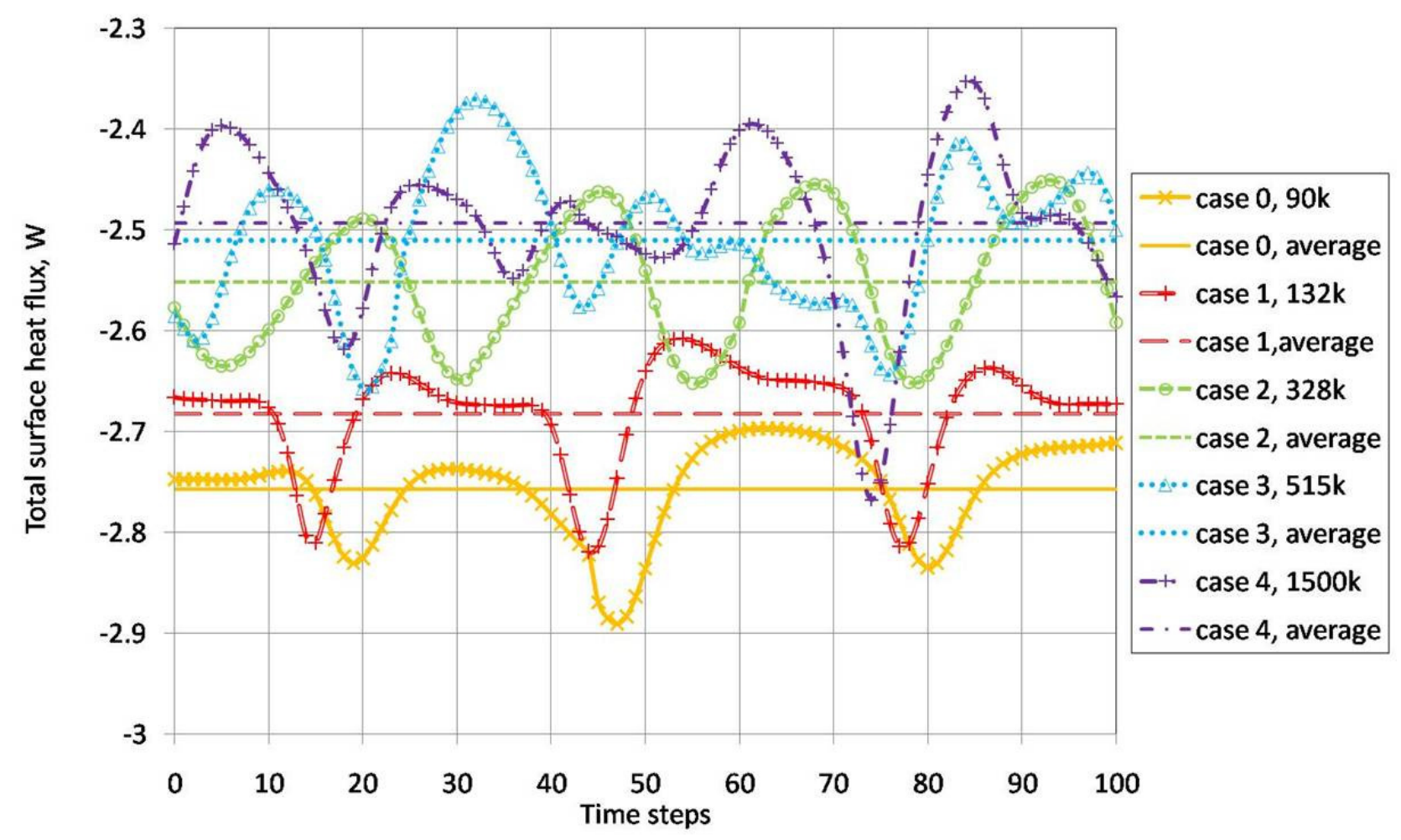

(b) Changes of the average temperatures of the mattress during calculations

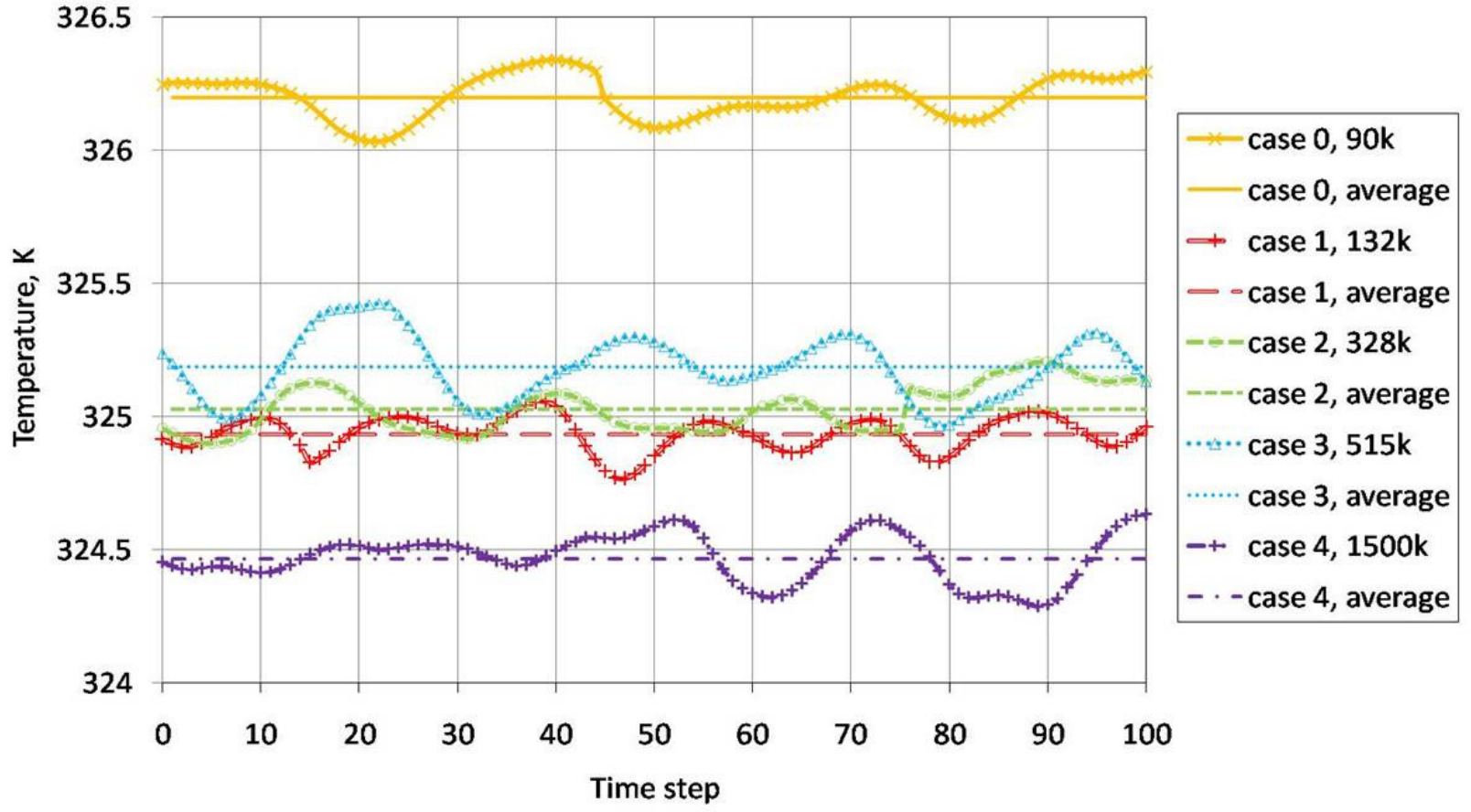

Figure 3.2. The variations in time of the average value for (a) the total heat transfer rate on the infant skin, and (b) the average temperature on the mattress for the five meshes investigated. 
(a)

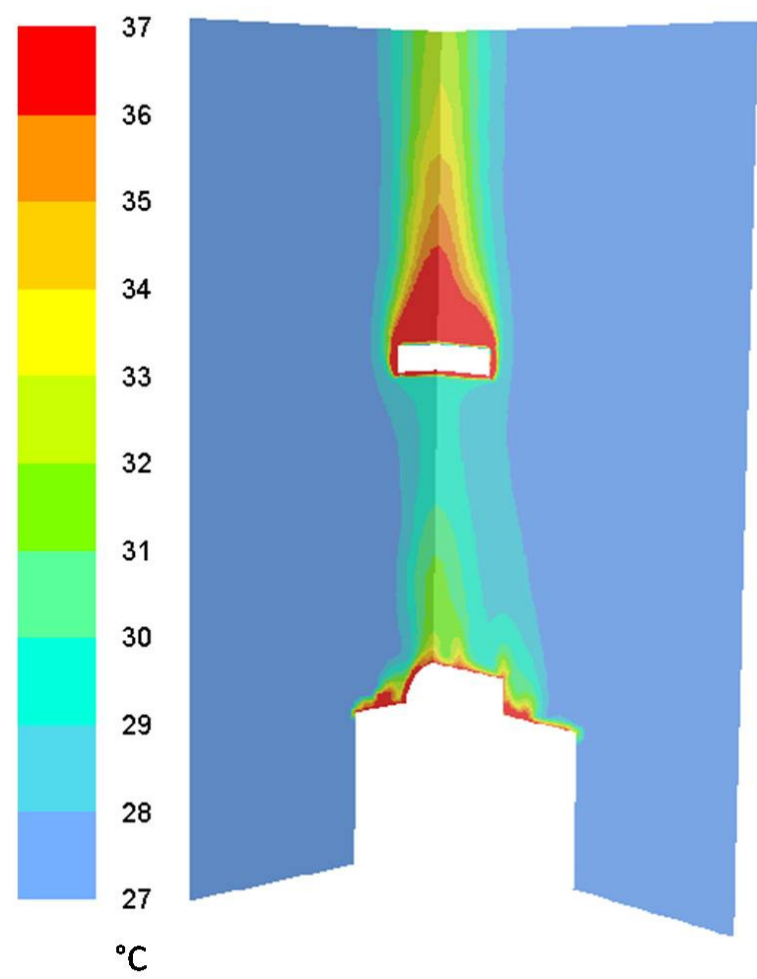

(b)

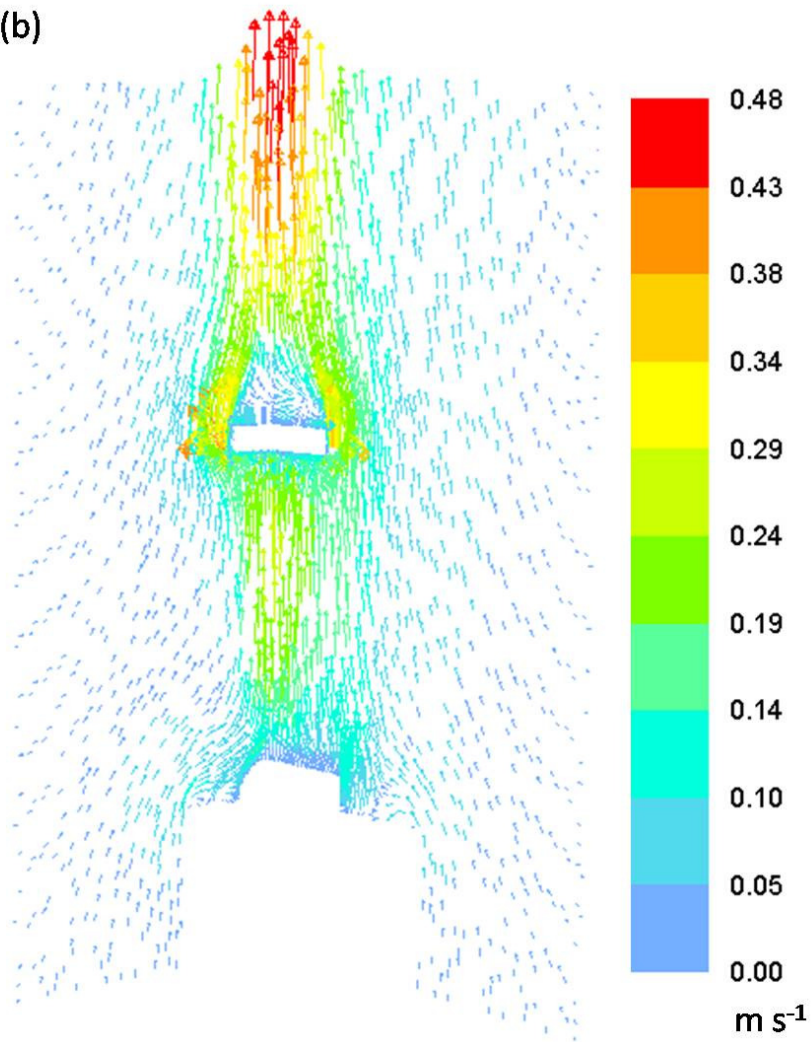

Figure 3.3. (a) The temperature distribution, and (b) the velocity vectors, plotted on the symmetry planes of the radiant warmer.

(a)

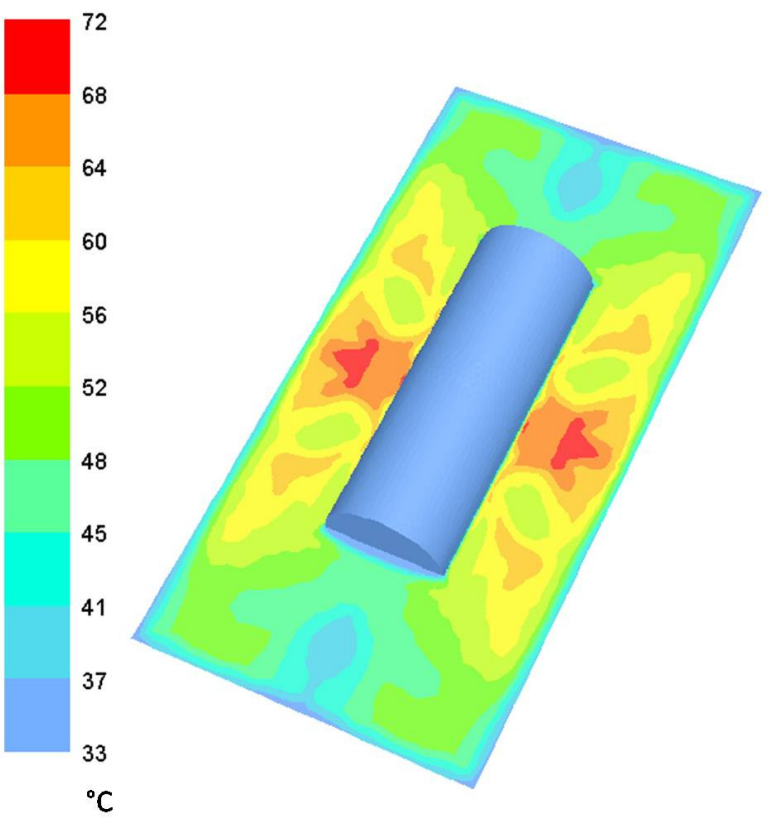

(b)

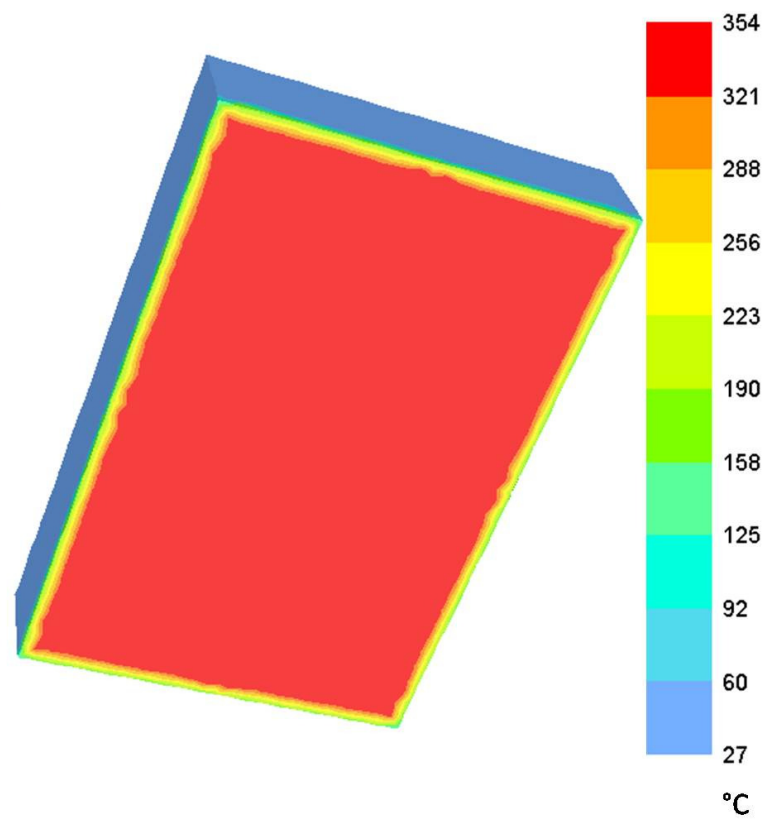

Figure 3.4. The temperature distribution (a) on the mattress, and (b) on the surface with the heat source. 
Table 2.1

Number of Elements within each Grid.

\begin{tabular}{cc}
\hline Name of the case & Number of elements \\
\hline Case 0 & $90 \mathrm{k}$ \\
Case 1 & $132 \mathrm{k}$ \\
Case 2 & $328 \mathrm{k}$ \\
Case 3 & $515 \mathrm{k}$ \\
Case 4 & $1500 \mathrm{k}$ \\
\hline
\end{tabular}

Table 2.2

Thermal Properties of the Air.

\begin{tabular}{cc}
\hline Name of the Property & Value Prescribed \\
\hline Specific heat $\left(\mathrm{J} \mathrm{kg}^{-1} \mathrm{~K}^{-1}\right)$ & 1006.430 \\
Thermal conductivity $\left(\mathrm{W} \mathrm{m}^{-1} \mathrm{~K}^{-1}\right)$ & 0.026 \\
Kinematic viscosity $\left(\mathrm{m}^{2} \mathrm{~s}^{-1} \times 10^{-6}\right)$ & 17.890 \\
\hline
\end{tabular}

Table 3.1

The Heat Transfer Rate Balance on the Major Surfaces in the Model.

\begin{tabular}{lcc}
\hline \multirow{2}{*}{ Name of the Surface } & $\begin{array}{c}\text { Total Surface Heat } \\
\text { Transfer Rate }(\mathrm{W})\end{array}$ & $\begin{array}{c}\text { Radiation Heat } \\
\text { Transfer Rate (W) }\end{array}$ \\
\hline Baby & -2.6 & -3.0 \\
Mattress & 0.0 & -6.5 \\
Radiant source & 150.0 & 120.0 \\
\hline
\end{tabular}

Table 3.2

Radiation Heat Transfer Rate on the Inlets in the Model.

\begin{tabular}{lc}
\hline Name of the Surface & $\begin{array}{c}\text { Radiation Heat } \\
\text { Transfer Rate }(\mathrm{W})\end{array}$ \\
\hline Back Inlet & -29.6 \\
Right Inlet & -55.0 \\
Bottom Inlet & -23.9 \\
Top outlet & -2.3 \\
\hline
\end{tabular}

